\title{
Engineering terahertz surface magnon-polaritons in hyperbolic antiferromagnets
}

\author{
Rair Macêdo ${ }^{1, *}$ and Robert E. Camley ${ }^{2}$ \\ ${ }^{1}$ SUPA School of Physics and Astronomy, University of Glasgow, Glasgow G12 8QQ, United Kingdom \\ ${ }^{2}$ Center for Magnetism and Magnetic Nanostructures, University of Colorado at Colorado Springs, Colorado Springs, Colorado 80918, USA
}

(Received 17 October 2018; revised manuscript received 8 January 2019; published 29 January 2019)

\begin{abstract}
Magnetic crystals were recently studied as a route to hyperbolic dispersion and the effects associated with it. These studies, however, concentrated on bulk waves and frequencies where transmission is possible and where negative refraction occurs. Here, in contrast, we concentrate on geometries which sample regions of the dispersion relations where bulk propagation is not possible. This is done by controlling the orientation of the uniaxial anisotropy axis with respect to the surface of the crystal. Furthermore, we find that new magnetic surface polaritons exist in these regions, and we investigate the nature of these waves. In addition, significant tunability can be introduced by applying an external field perpendicular to the easy axes of a uniaxial antiferromagnet, creating a canted structure and generally shifting the frequencies to higher values. This externally applied field dramatically changes the nature of both surface and bulk polaritons, making them highly nonreciprocal.
\end{abstract}

DOI: 10.1103/PhysRevB.99.014437

\section{INTRODUCTION}

In recent years, we have witnessed increased efforts towards engineering novel structures to control and manipulate electromagnetic waves. Metamaterials, for example, which were initially engineered to display negative refraction [1], have recently received significant attention in the context of unusual surface polaritons [2]. This has led to the design of new types of metamaterials which not only support but also guide such waves [3-6]. For example, van der Waals heterostructures [7-10] that have hyperbolic dispersion [6,11] display interesting features in their surface polaritons. Similar phenomena are also observed in man-made structures, often referred to as hyperbolic metamaterials. These structures are engineered so that not all the diagonal components of the permeability or permittivity tensor have the same sign at a given frequency [12]. These are, however, a subclass of anisotropic media which have, for decades, been associated with surface polaritons [13]. Understanding and controlling the characteristics of these waves in anisotropic dielectric and/or magnetic media has been, also for many years, the topic of research worldwide.

A well-known example of anisotropic materials displaying unusual surface polaritons is that of antiferromagnets [14-17]. For example, waves propagating on the surface of an antiferromagnetic crystal can be nonreciprocal, where reversal of the wave vector $k$ leads to a new frequency $\omega$, i.e., $\omega(k) \neq$ $\omega(-k)$. This nonreciprocity occurs when the symmetry of the system is broken, thus producing a surface mode that depends on the direction of the wave [16]. Much of what is known about these nonreciprocal polaritons, however, has been investigated in the classic Voigt geometry where the hyperbolic behavior does not play a role.

*Rair.Macedo@glasgow.ac.uk
In this work, we investigate the behavior of surface magnon-polaritons in canted antiferromagnetic crystals acting as hyperbolic media. These media were recently used to study negative refraction [18] and focusing by flat lenses [19] due to hyperbolic dispersion. In these studies, the easy axis of the antiferromagnet was parallel to the surface, which allows bulk waves to propagate throughout the entire hyperbolic frequency range. In this paper, in contrast, we consider different geometries in which the easy axis may be rotated with respect to the surface. This rotation has a number of important consequences:

(i) The orientation of the anisotropy axis can be used to control the allowed wave vectors for bulk waves in the antiferromagnet.

(ii) Changing the easy axis direction changes the frequency and wave vector regions where bulk wave propagation parallel to the surface is forbidden.

(iii) We find that new magnetic surface polaritons exist in these forbidden regions.

(iv) A static magnetic field applied parallel to the surface, but perpendicular to the easy axis, increases the frequency width of the forbidden regions and induces great nonreciprocity for both surface and bulk polaritons.

\section{CONSIDERATIONS ON THE NATURE OF THE PERMEABILITY TENSOR}

Surface magnon-polaritons are directly associated with the permeability tensor of magnetic media. In antiferromagnets, the electromagnetic fields of the incident radiation couple with the spin precession, and the magnetic permeability tensor varies rapidly with frequency close to the fundamental magnetic resonances of the material, usually in the terahertz region [17]. 
(a)
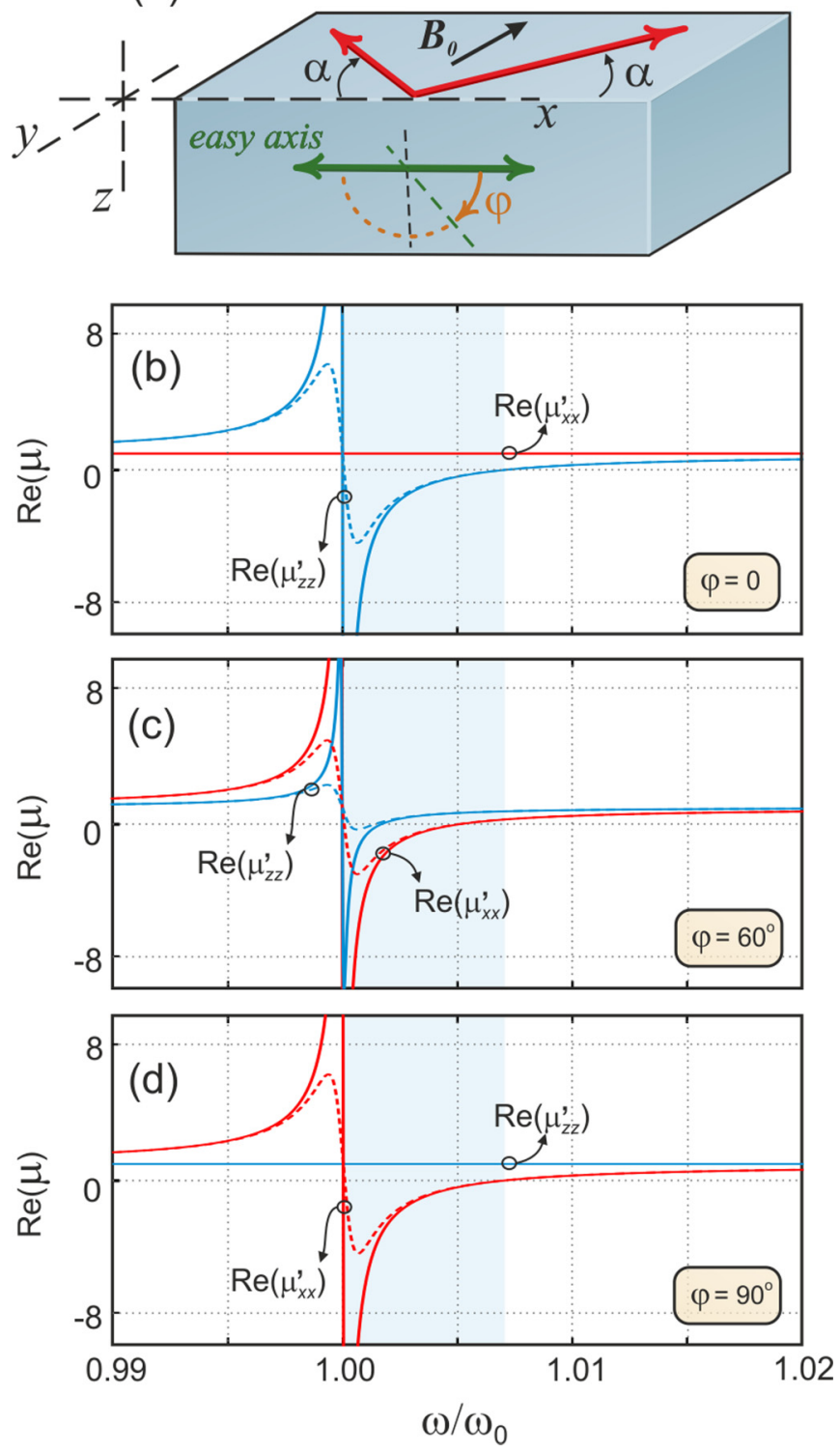

FIG. 1. (a) Geometry showing the rotation of the easy axis in the $x z$ plane given by the angle $\varphi$. The spin canting, due to the field $\mathbf{B}_{\mathbf{0}}$ externally applied along $y$, is depicted by the red arrows and can be quantified by the angle $\alpha$. Diagonal components of the permeability tensor as a function of frequency for $B_{0}=0$ calculated for rotation angles of (b) $\varphi=0$, (c) $\varphi=60^{\circ}$, and (d) $\varphi=90^{\circ}$. The shaded region shows the frequency range wherein hyperbolic behavior is found. Solid lines are for $T=0.06 T_{N}$, and dashed lines are for $T=0.42 T_{N}$.

Our geometry is illustrated in Fig. 1(a). The $x y$ plane defines the surface of the crystal. The anisotropy axis can be rotated in the $x z$ plane by an angle $\varphi$, where $\varphi=0$ indicates an anisotropy axis parallel to the surface and $\varphi=\pi / 2$ indicates that the anisotropy axis is perpendicular to the surface. If a magnetic field is present, it is applied parallel to the surface and along $y$, thus causing a canted magnetic configuration.

We start by investigating the behavior of the permeability tensor in its simplest form, which is in the absence of an applied magnetic field $\mathbf{B}_{\mathbf{0}}$. In this case, the equilibrium magnetizations of all sublattices are perfectly aligned antiparallel to one another. If we consider the alignment direction, i.e., the easy axis, to be $x$, the permeability tensor component $\mu_{x x}$ is unity at all frequencies. The motion of the spins at the antiferromagnetic resonance is confined to the $y z$ plane, and $\mu_{y y}=\mu_{z z}$. The permeability has resonance poles at the antiferromagnetic frequency [20]:

$$
\omega_{0}^{2}=\gamma^{2}\left(2 B_{A} B_{E}+B_{A}^{2}\right),
$$

where $\gamma$ is the gyromagnetic ratio. Here, $B_{A}$ measures the anisotropy field, and $B_{E}$ is the exchange field exerted on a spin in one sublattice by the neighboring spins in the other sublattice of the antiferromagnet. These are the effective fields constraining the sublattice magnetizations to the preferred axis (here taken to be $+x$ and $-x$ ) and are antiparallel to one another.

The resonant behavior becomes much more complicated when a magnetic field is externally applied as antiferromagnets become gyrotropic, i.e., the permeability tensor has offdiagonal elements. In the present case, we consider an external field $\mathbf{B}_{\mathbf{0}}$ to be applied perpendicular to the spin alignment direction (along $y$ ) so that the spins cant towards the field direction, as shown in Fig. 1(a). The spin canting is quantified by an angle $\alpha$ which is a function of the aforementioned fields $[19,21,22]$. The resonance frequency now becomes

$$
\omega_{\perp}^{2}=\omega_{0}^{2} \cos ^{2} \alpha+2 \gamma^{2} B_{0} B_{E} \sin \alpha .
$$

This spin canting also makes $\mu_{y y} \neq \mu_{z z}$, as $\mu_{y y}$ is now affected by a weak ferromagnetism along the external field direction, and $\mu_{x x}$ is no longer unity. The permeability tensor now has the form [21]

$$
\overleftrightarrow{\mu}(\omega)=\left[\begin{array}{ccc}
\mu_{x x} & 0 & \mu_{x z} \\
0 & \mu_{y y} & 0 \\
-\mu_{x z} & 0 & \mu_{z z}
\end{array}\right] .
$$

In these materials, the condition for hyperbolic behavior, that the diagonal components of the permeability tensor should possess opposing signs, can easily be met near the magnonpolariton resonance frequencies [19].

The nature of hyperbolic magnon-polaritons, particularly surface magnon-polaritons (SMPs) in antiferromagnets, however, can be changed by choosing a different orientation for the uniaxial anisotropy with respect to the crystal's surface. Here, we model this as a rotation of the easy axis, so that the surface of the crystal is always in the $x y$ plane, but the easy axis is effectively allowed to rotate in the $x z$ plane, as shown in Fig. 1(a). The permeability in the fixed coordinate system can now be obtained by a crystal axis transformation, introducing a rotation along the $x z$ plane given by the angle $\varphi$. If a rotation takes place $(\varphi \neq 0)$, the components of the permeability tensors given in Eq. (3) have to be rewritten in terms of the angle of rotation $\varphi$ and are now given by

$$
\begin{aligned}
& \mu_{x x}^{\prime}=\mu_{x x} \cos ^{2} \varphi+\mu_{z z} \sin ^{2} \varphi, \\
& \mu_{z z}^{\prime}=\mu_{z z} \cos ^{2} \varphi+\mu_{x x} \sin ^{2} \varphi, \\
& \mu_{x z}^{\prime}=\mu_{x z}+\left(\mu_{z z}-\mu_{x x}\right) \cos \varphi \sin \varphi, \\
& \mu_{z x}^{\prime}=-\mu_{x z}+\left(\mu_{z z}-\mu_{x x}\right) \cos \varphi \sin \varphi .
\end{aligned}
$$


Such a tensor is obtained using $\overleftrightarrow{\mu^{\prime}}(\omega)=\mathbf{T} \overleftrightarrow{\mu}(\omega) \mathbf{T}^{-1}$, where $\mathbf{T}$ is the transformation matrix in the $x z$ plane and $\mathbf{T}^{-\mathbf{1}}$ is its transpose. The expressions for the components of $\overleftrightarrow{\mu}(\omega)$, which is the original permeability tensor given in Eq. (3), are given explicitly in Appendix A, and the full derivation can be found in Refs. [19,21].

As an example, in Figs. 1(b)-1(d), we plot two diagonal components of the permeability tensor, $\overleftrightarrow{\mu^{\prime}}(\omega)$, given in Eq. (4) as a function of frequency and for different rotation angles. We use the example material $\mathrm{MnF}_{2}$, which is a wellcharacterized antiferromagnet below its Néel temperature, $T_{N}$, of $67 \mathrm{~K}$. At a temperature of $4.2 \mathrm{~K}$ its relevant parameters are the sublattice magnetization $M_{S}=6.0 \times 10^{5} \mathrm{~A} / \mathrm{m}$, anisotropy field $B_{A}=0.787 \mathrm{~T}$, and exchange field $B_{E}=53.0$ $\mathrm{T}[23]$.

In Figs. 1(b)-1(d), we also highlight the behavior of the permeability for different temperatures. In order to model this, we use an approach similar to that done by Remer and coworkers [23], in which the temperature change leads to changes in the damping and in the gyromagnetic ratio. At $T=$ $0.06 T_{N}$ we have $\Gamma / \omega_{0}=8 \times 10^{-5}$, and at $T=0.42 T_{N}$ we have $\Gamma / \omega_{0}=6.5 \times 10^{-4}$. Similarly, $\gamma / 2 \pi c=0.928 \mathrm{~cm}^{-1} / \mathrm{T}$ at $T=0.06 T_{N}$, and $\gamma / 2 \pi c=0877 \mathrm{~cm}^{-1} / \mathrm{T}$ for $T=0.42 T_{N}$. The former case corresponds to a resonance frequency of $\omega_{0} / 2 \pi c=8.67 \mathrm{~cm}^{-1}$ (or $0.26 \mathrm{THz}$ ), while the latter has a resonance frequency of $\omega_{0} / 2 \pi c=8.19 \mathrm{~cm}^{-1}$ (or $0.24 \mathrm{THz}$ ).

Upon examining the permeability tensor components given in Fig. 1(b), when there is no rotation of the anisotropy axis, we can see that near the magnetic resonance $\mu_{z z}^{\prime}<0$ and $\mu_{x x}^{\prime}>0$ [19]. If we then consider transverse-electric- (TE) polarized radiation propagating in the $x z$ plane, we find that the diagonal permeability tensor component perpendicular to the surface, $\mu_{z z}^{\prime}$, is negative and the component parallel to the surface, $\mu_{x x}^{\prime}$, is positive. In the absence of damping $(\Gamma=0)$, this allows propagation of bulk waves for (along $z$ ) all wave vectors $k_{\|}$. However, in practice propagation of large $k_{\|}$'s may be impaired according to the damping of the materials, as discussed by Othman et al. [24] for graphenebased hyperbolic metamaterials and Macêdo et al. [19] for antiferromagnetic slab lensing.

In Fig. 1(c) we show the same components when a $60^{\circ}$ rotation of the anisotropy axis takes place. The introduction of a nonzero $\varphi$ results in a rotation of the hyperbolic isofrequency curves [25]. This is due to the off-diagonal components of $\overleftrightarrow{\mu^{\prime}}(\omega)$, which now possess a real part even at zero damping $(\Gamma=0)$, thus contributing to the regions where bulk waves are (or are not) allowed to propagate. This has a significant impact on the nature of the surface polaritons, as we will see in the following sections.

Finally, we consider the easy axis rotated by $90_{\leftrightarrow}^{\circ}$, i.e., anisotropy directly along $z$, so that the component of $\stackrel{\leftrightarrow}{\mu^{\prime}}(\omega)$ along $x$ is now negative and the one along $z$ is positive, as shown in Fig. 1(d). This means that propagation of bulk waves (along $z$ ) in the hyperbolic frequency region is forbidden for small wave vectors $k_{\|}$but allowed for large ones [25,26].

Now that we understand the anisotropic behavior of antiferromagnetic media we move to the problem of surface magnon-polaritons and how to detect them.

\section{SURFACE POLARITONS}

Surface polaritons are waves propagating along the crystal's surface, typically with an exponential decay of amplitude away from the surface. As noted earlier, these modes have been studied both experimentally $[27,28]$ and theoretically [15] but mostly in the limited geometry in which the anisotropy axis is parallel to the surface. At the surface of an antiferromagnetic crystal, as depicted in Fig. 1(a), these waves couple with TE-polarized light, i.e., electric field constrained to the $y$ direction.

Here, we study whether surface waves are allowed in other geometries. We assume surface-wave solutions with propagation along $x$ and decay along $z$ (given by the parameters $\beta_{0}$ and $\beta$ ). These solutions can be written as

$$
\mathbf{E}(x, z, t)=\hat{y} E e^{i\left(k_{\|} x-\omega t\right)} e^{\beta_{0} z}
$$

and

$$
\mathbf{E}(x, z, t)=\hat{y} E e^{i\left(k_{\|} x-\omega t\right)} e^{-\beta z}
$$

for air and the antiferromagnet, respectively. Using Eqs. (5) and (6), we can find equations for the decay parameters through Maxwell's equations (the full calculations are given in Appendix B). The boundary conditions then allow us to match the solutions inside and outside the material to find the following implicit dispersion relation for the surface polaritons:

$$
\beta_{0}+\frac{\beta \mu_{z z}^{\prime}-i k_{\|} \mu_{x z}^{\prime}}{\mu_{x x}^{\prime} \mu_{z z}^{\prime}-\mu_{x z}^{\prime} \mu_{z x}^{\prime}}=0 .
$$

In the special cases when the anisotropy axis lies either directly along the $x$ or $z$ direction (corresponding to $\varphi=0$ or $\varphi=90^{\circ}$, respectively) and in the absence of an externally applied field, so that the off-diagonal components of $\overleftrightarrow{\mu^{\prime}}(\omega)$ vanish, Eq. (7) is reduced to

$$
\beta_{0}+\frac{\beta}{\mu_{x x}^{\prime}}=0 .
$$

Since both $\beta_{0}$ and $\beta$ are positive, Eq. (8) can be solved only when $\mu_{x x}^{\prime}$ is negative. This is, therefore, the condition for the existence of surface polaritons, and it can be met only when the anisotropy axis lies along the $z$ direction, so that $\mu_{x x}^{\prime}=$ $\mu_{z z}$. This can be easily evaluated to find $k_{\|}$as a function of $\omega$ by replacing the values of $\beta_{0}$ and $\beta$ (found in Appendix $\mathrm{B}$ ) in Eq. (8), yielding the following implicit dispersion relation:

$$
\frac{c^{2}}{\omega^{2}} k_{\|}^{2}=\frac{\varepsilon-\mu_{z z}}{1-\mu_{z z}} .
$$

In its general form, however, Eq. (7) must be solved numerically, which can be troublesome, especially when the surface waves are confined to a narrow frequency region. Here, however, we employ a simpler technique in order to visualize the dispersion relation. We plot the logarithm of the absolute value of the left side of Eq. (7) as a function of both $k_{\|}$ and $\omega$ and look for zeros; the results are shown in Fig. 2, where white dots indicate a value of zero and hence the existence of a surface mode.

We comment on some of the features in Fig. 2 and focus on the frequency range where hyperbolic behavior is seen 


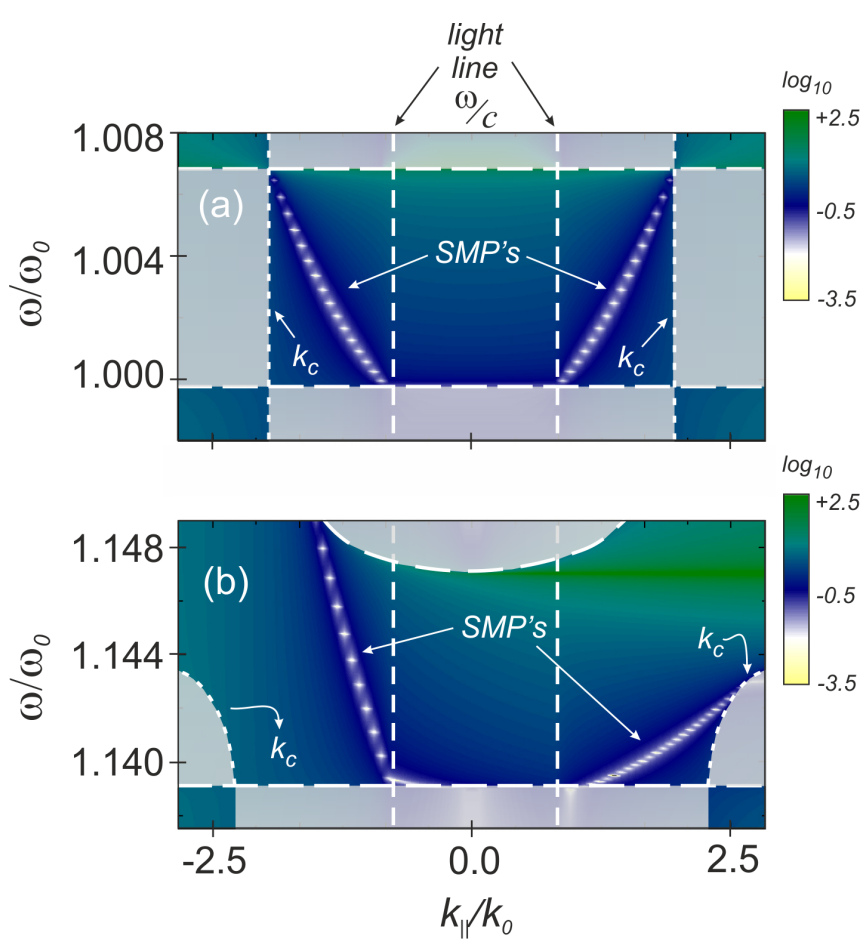

FIG. 2. Dispersion relation of the surface magnon-polaritons (SMPs) from Eq. (2) in a geometry in which the anisotropy axis is perpendicular to the crystal's surface $\left(\varphi=90^{\circ}\right)$. Examples for (a) $B_{0}=0$ and (b) $B_{0}=5 \mathrm{~T}$. The surface modes are identified as the bright white regions, and the regions where bulk modes are allowed to propagate are highlighted by the added gray shading. Note that when Eq. (7) $=0$, its logarithm is undefined. For this reason, we have removed all exact zeros from the solution, and the bright spots are composed only by Eq. (7) $\rightarrow 0$.

(in the range $1.0<\frac{\omega}{\omega_{0}}<1.007$ ). As discussed earlier, bulk polaritons in antiferromagnetic crystals when $\varphi=0$ can propagate for every wave vector $k_{\|}$. In that case and when there is no externally applied magnetic field, there is no bulk wave forbidden region at $k_{\|}=0$; the upper and lower bulk bands touch each other, and no surface polaritons are observed.

However, as we see in Fig. 2, surface polaritons can be created, even in the absence of an external field, by simply rotating the crystal's easy axes in the $x z$ plane. (Of course, this really is not done by rotating the easy axis; instead, one can cut the crystal along different planes to obtain surfaces at a given angle with respect to the easy axis.) In Fig. 2 we show the dispersion relation of surface magnon-polaritons when $\varphi=$ $90^{\circ}$ in the absence of an external field [Fig. 2(a)] and at an externally applied field of $5 \mathrm{~T}$ [Fig. 2(b)].

As discussed earlier, if $\mu_{x x}^{\prime}$ is negative, bulk propagation is prohibited for low wave vectors $k_{\|}$. This condition holds for modes with wave vectors $\left|k_{\|}\right| \leqslant k_{c}$, where $k_{c}^{2}=\varepsilon_{3} \mu_{z z}^{\prime} k_{0}^{2}$ is the cutoff transverse spatial frequency [26] and $k_{0}=\omega / c$. This is shown by the shading in Fig. 2. Modes with transverse wave vectors located within the band gap $-k_{c}<k_{\|}<+k_{c}$ decay evanescently in the $z$ direction, as required for surface polaritons.

In the absence of an external field, these surface magnon polaritons are reciprocal and extend in frequency from the upper bulk band down to the lower bulk band [see Fig. 2(a)].
This behavior, however, is highly disturbed when an external field is applied where the surface polaritons become highly nonreciprocal, as seen in Fig. 2(b). In addition, the group velocity $v_{g}=\frac{d \omega}{d k_{\|}}$of the surface polaritons is also highly nonreciprocal. These factors lead to the possibility of developing infrared isolators or other signal-processing devices. Here, we have concentrated on natural structures, which require large fields in order to achieve significant tunability. However, similar behavior should be expected in recently investigated artificial structures based on the same principles [29].

\section{ATTENUATED TOTAL REFLECTION}

We now examine whether the new surface polaritons discussed above could be seen experimentally. For this, we consider an attenuated total reflection (ATR) geometry in the classic Otto configuration. This is a three-layered system consisting of a dielectric prism, an air gap, and an antiferromagnetic sample, as shown in Fig. 3.

We choose the $z$ axis to be normal to the interfaces and the $x z$ plane to be the plane of incidence; an external magnetic field $\mathbf{B}_{\mathbf{0}}$ is applied perpendicular to both the spin orientation direction and the plane of incidence (along $y$ ), consistent with the permeability tensor given by Eq. (4) $(\varphi \neq 0)$. The air gap extends from $z=-d$ to $z=0$.

The prism is chosen with a relatively large refractive index so that the dielectric constant of the prism is greater than the dielectric constant of air $\left(\varepsilon_{p}>\varepsilon_{2}\right)$, and the angle of incidence $\theta_{1}$ is greater than the critical angle for total internal reflection. Thus, if the third layer is absent, the incident light is totally reflected. However, total internal reflection requires the presence of an evanescent wave with decreasing amplitude in the air gap. This wave can excite surface waves in the antiferromagnetic surface, which removes energy and thus produces an attenuation of the total reflection spectra.

In order to look at the behavior of these waves and their ATR spectra, we start by considering a TE-polarized beam incident at the antiferromagnetic surface with a frequency $\omega$ so that its wave vector $\mathbf{k}=\left(\hat{x} k_{\|}, 0, \hat{z} k_{\perp}\right)$. The component of

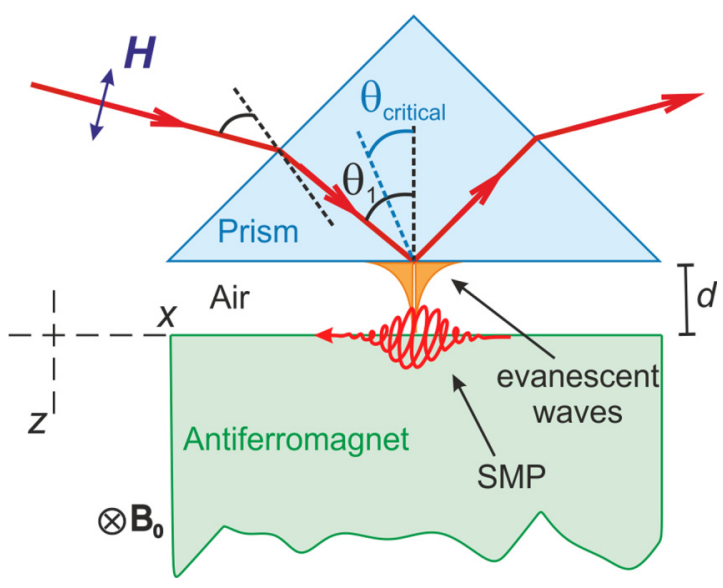

FIG. 3. Geometry of attenuated total reflection off an antiferromagnetic crystal's surface. Here, we consider only TE-polarized incident waves, so that the magnetic field of the radiation lies on the $x z$ plane. We allow the crystal's easy axis to rotate by an angle $\varphi$ along the $x z$ plane, as shown in Fig. 1(a). 
the wave vector parallel to the surface $k_{\|}$is given by

$$
k_{\|}=\frac{\omega}{c} \sqrt{\varepsilon_{p}} \sin \theta_{1} .
$$

Standard boundary conditions require the fields to be continuous at the boundaries so that $k_{\|}$does not change across the layers. The wave vector component perpendicular to the surface $k_{\perp}$, however, changes according to the medium in which the wave is propagating. We can employ Maxwell's equations to find the component of the wave vector perpendicular to the surface for each medium. The dispersion relation in each medium gives

$$
\begin{aligned}
& k_{\perp 1}=\frac{\omega}{c} \sqrt{\varepsilon_{p}} \cos \theta_{1}, \\
& k_{\perp 2}^{2}+k_{\|}^{2}=\frac{\omega^{2}}{c^{2}} \sqrt{\varepsilon_{2}},
\end{aligned}
$$

and

$$
\begin{gathered}
\mu_{z z}^{\prime} k_{3 \perp}^{2}+\mu_{x x}^{\prime} k_{\|}^{2}+k_{\|} k_{3 \perp}\left(\mu_{x z}^{\prime}+\mu_{z x}^{\prime}\right) \\
=\frac{\omega^{2}}{c^{2}} \varepsilon_{3}\left(\mu_{x x}^{\prime} \mu_{z z}^{\prime}-\mu_{x z}^{\prime} \mu_{z x}^{\prime}\right),
\end{gathered}
$$

where the subscripts represent the prism (1), air (2), and antiferromagnet (3).

Note that the relation given by Eq. (13) is substantially different for that given elsewhere for canted antiferromagnets $[19,22]$. This comes as a consequence of the easy-axis rotation shown in Fig. 1(a) and the nature of the permeability tensor given by Eq. (4), where the off-diagonal components are different from each other, as discussed earlier.

The form of these wave vectors is the basis for the calculation of the ATR reflection coefficient, which can be done through a standard technique given elsewhere [15]. A short derivation of the calculation for the ATR reflectivity is given in Appendix C. The ATR technique is particularly useful for detecting surface waves, which are usually confined to regions where $k_{\|}^{2}>\frac{\omega}{c} \sqrt{\varepsilon_{2}}$, leading to exponential decay or an increase in the gap region. This means that surface waves cannot be excited by a light beam incident directly from air into the antiferromagnetic surface as such a beam satisfies the condition given in Eq. (12) with real $k_{\perp 2}$. However, with sufficiently large $\varepsilon_{p}$ and $\theta_{1}$, as achieved in ATR measurements, the condition necessary to excite a surface mode at the antiferromagnetic surface can be met.

\section{RESULTS}

In this section, we present some examples for the behavior of bulk and surface polaritons as seen through ATR reflection maps. We examine reflection spectra in cases with and without an external field and also show the effect of temperature.

\section{A. Surface polaritons in the absence of a magnetic field}

To visualize and understand the behavior of polaritons such as the ones so far investigated through analytic solutions, we make use of reflection maps. These are plots of ATR reflectivity as a function of both $k_{\|}$and the frequency of the incident wave $\omega$. This method was recently used by Anderson and Camley to probe surface and bulk polaritons in hexagonal ferrites [30]. In Fig. 2(a) we have already presented an example of the surface polaritons at $\varphi=90^{\circ}$. In Fig. 4 we show reflection maps for various rotation angles $\varphi$, showing the evolution of the surface polaritons with angle for the case where there is no applied field. Here, both bulk and surface magnon-polaritons are clearly seen. However, because attenuated total reflection does not couple strongly with bulk modes, especially for large $k_{\|}$, we show the boundaries of the bulk bands using the white grid.

For the first case [Fig. 4(a)], where $\varphi=30^{\circ}$, the surface and bulk polaritons are almost indistinguishable. However, as $\varphi$ increases and the gap regions where bulk waves are forbidden become wider, the surface polaritons become easier to distinguish, as shown in Fig. 4(b) for $\varphi=60^{\circ}$.

For the case where the easy axis lies perpendicular to the surface $\left(\varphi=90^{\circ}\right)$, shown in Fig. 4(c), a wide forbidden region for bulk propagation is seen. In these regions, reflection is always maximal except for where surface modes exist. These are seen going from both sides of the upper bulk band all the
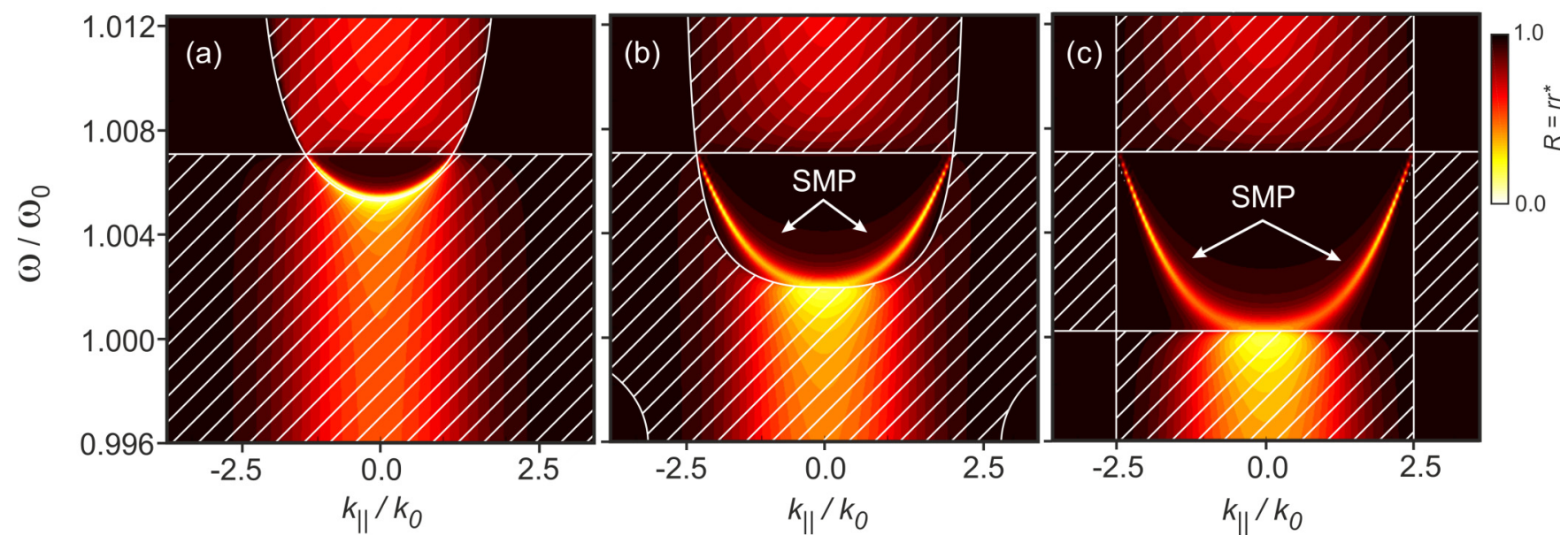

FIG. 4. Calculated ATR maps showing how SMP's can be dramatically modified by the angle between the crystal's easy axis and the crystal's surface, $\varphi$. The reflection maps are calculated for a temperature $T=0.04 T_{N}$ and rotation angles (a) $\varphi=30^{\circ}$, (b) $\varphi=60^{\circ}$, and (c) $\varphi=90^{\circ}$. We based our calculations on a prism with a dielectric constant of $\varepsilon_{p}=11.56(\mathrm{Si})$, and the dielectric constant of $\mathrm{MnF}_{2}$ was taken to be $\varepsilon_{3}=5.5$. The applied field is zero in this case, and the waves are reciprocal. 


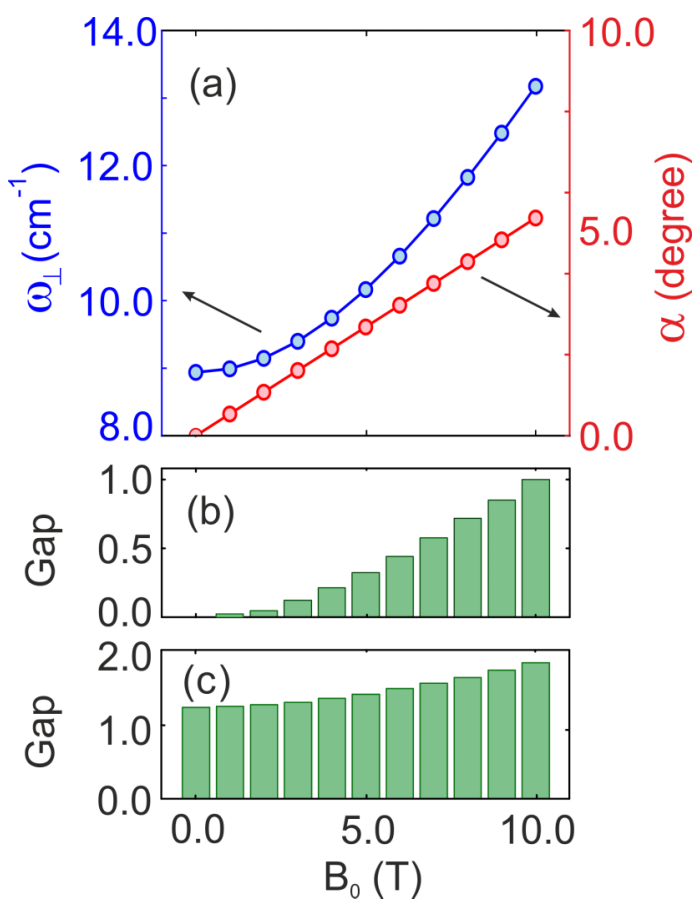

FIG. 5. (a) Resonance frequency $\omega_{r}$ and canting angle $\alpha$ as a function of the externally applied field $\mathbf{B}_{\mathbf{0}}$. Width of the frequency gap forbidden for bulk waves as a function of the external field measured at $k_{\|}=0$ and (b) $\varphi=0$ and (c) $\varphi=90^{\circ}$. Note that (b) and (c) were normalised to the width of the frequency gap of $\varphi=0$ with $B_{0}=10 \mathrm{~T}$.

way across the forbidden bulk region and falling into the lower bulk band, in great agreement with the analytic solution shown in Fig. 2(a).

\section{B. Field tunability and nonreciprocity}

Up until now, our results have shown only the effects of rotating the crystal's easy axis. If there is no externally applied field, the system is still symmetric. In this case and as seen from Fig. 2(a), the surface polaritons are reciprocal. In the case where an external field is applied perpendicular to the spin alignment direction to induce spin canting, the nature of these surface polaritons dramatically changes, as we will see. In order to discuss the effect of an external field on the surface polaritons we start by looking at the spin canting induced by the field, as shown in Fig. 5(a). As the external field increases, the resonance frequency $\omega_{r}$, calculated from Eq. (2), is shifted to higher values. It should be noted, also from Fig. 5(a), that even at high fields such as $10 \mathrm{~T}$ the canting angle is still very small (just over $5^{\circ}$ ). This is a consequence of the extremely high exchange fields preventing the spins from canting.

The external field not only acts a tuning mechanism but also induces a frequency gap region where bulk propagation is forbidden at normal incidence $\left(k_{\|}=0\right)$, as seen in Fig. 5(b) for $\varphi=0^{\circ}$. Note that we have normalized the width of the gap regions to that of $B_{0}=10 \mathrm{~T}$. However, even at the highest field used, the gap region is still not as wide as the one shown in Fig. 4(c) for $\varphi=90^{\circ}$ in the absence of an externally applied magnetic field. The effect of an external field on the gap width in this case $\left(\varphi=90^{\circ}\right)$ is given in Fig. 5(c), showing a steady increase of the gap.

Because the external field induces a large area of total reflection, even when there is no rotation of the easy axis, surface polaritons are possible in this geometry. In Fig. 6(a) and 6(b) we show not only how surface magnon-polaritons are induced by an external field but also how they are extremely nonreciprocal. In the case of $\varphi=0$, shown in Fig. 6(a), the attenuated total reflection map shows this surface polariton going all the way from one side of the upper bulk band down, influencing the other side of the lower bulk band. Thus, leading to a higher attenuation of the reflection for $+k_{\|}$when compared the reflection spectra of $-k_{\|}$.

In Fig. 6(b), we show the behavior of the surface magnonpolariton with an externally applied field of $5 \mathrm{~T}$ and with anisotropy axis at $\varphi=90^{\circ}$, i.e., with parameters equivalent to those for the analytic solution shown in Fig. 2(b). This case is of particular interest as it can also be compared to the case shown in Fig. 4(c). When an external field is applied, the surface polaritons shown earlier become nonreciprocal and leak energy into both the lower and upper bulk bands, leading to nonreciprocity for reflection involving bulk waves.
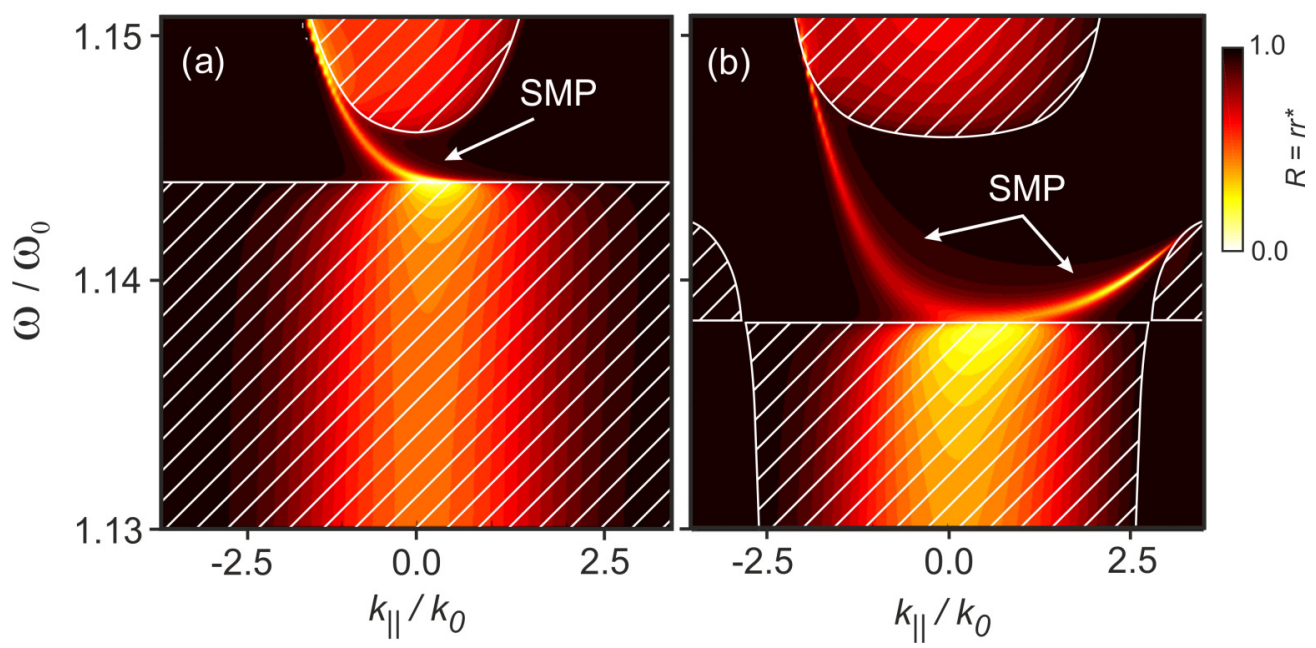

FIG. 6. Calculated ATR maps showing the nonreciprocal behavior of surface magnon-polaritons at $B_{0}=5$ T. Reflection maps calculated for a temperature $T=0.04 T_{N}$ and (a) $\varphi=0^{\circ}$ and (b) $\varphi=90^{\circ}$. 

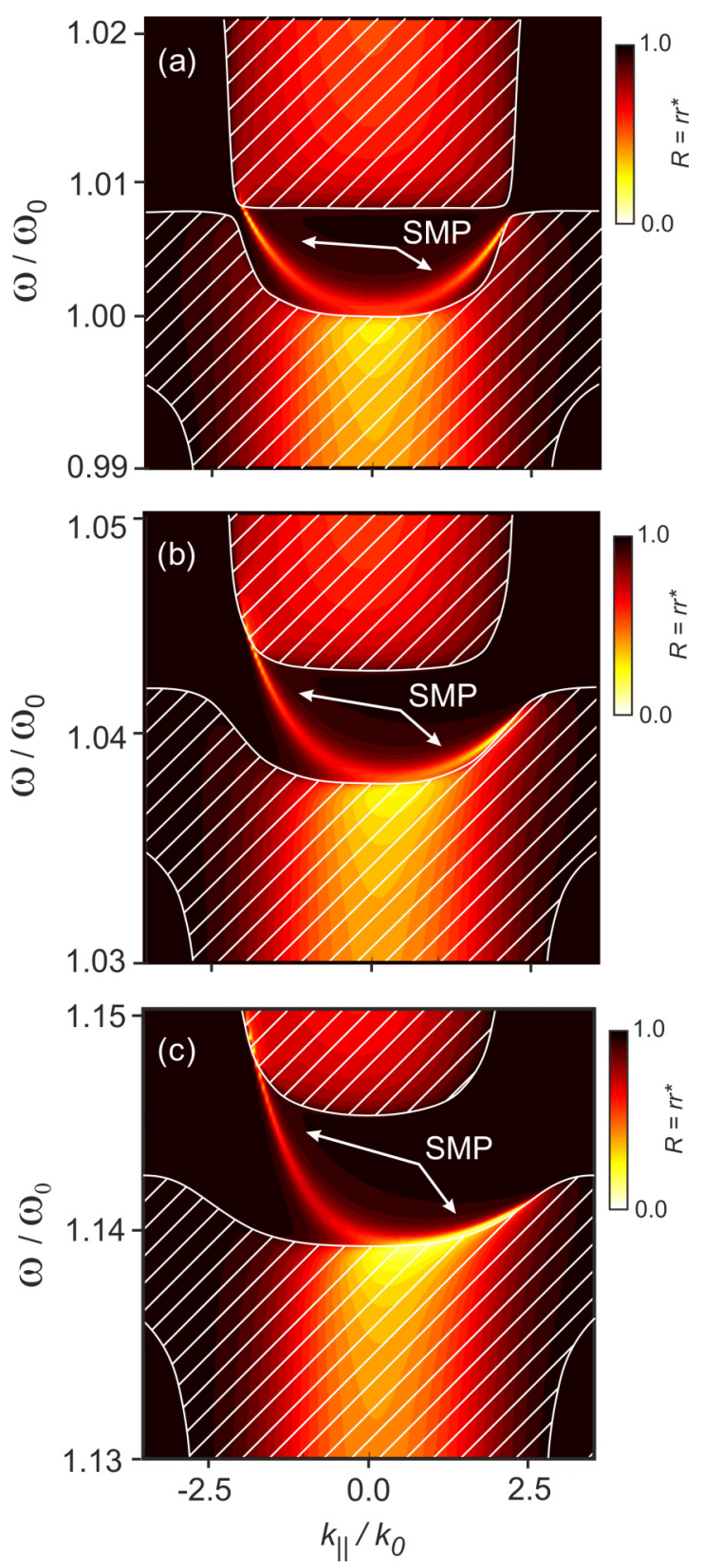

FIG. 7. ATR maps showing the evolution of surface magnonpolaritons for various externally applied field strengths (a) $B_{0}=$ $0.5 \mathrm{~T}$, (b) $B_{0}=2.5 \mathrm{~T}$, and (c) $B_{0}=5.0 \mathrm{~T}$. Reflection maps were calculated for a temperature $T=0.04 T_{N}$ and angle $\varphi=60^{\circ}$.

It is also interesting to look at the evolution of these surface magnon-polaritons as the field increases, as shown in Fig. 7. We have chosen to display the case of $\varphi=60^{\circ}$, where even the slightest externally applied field, given in Fig. 7(a), separates the upper and lower bulk bands and induces slight nonreciprocity of the surface mode. As the field increases, shown in Figs. 7(b) and 7(c), the bulk bands become much more separated, with a large area forbidden for bulk propagation wherein highly nonreciprocal surface magnon-polaritons are seen. Similar to the case shown in Fig. 6(b), the surface polariton leaks energy into the bulk bands, generally making the reflectivity extremely nonreciprocal so that $R\left(+k_{\|}\right)$is larger than $R\left(-k_{\|}\right)$for both bulk and surface modes.

\section{Temperature dependence}

The effect of temperature on the resonance frequency in antiferromagnetic media has been the subject of several studies. There has been some particular interest in the effect of temperature on reflections from antiferromagnetic surfaces, as discussed by Remer and coworkers [23]. Their work showed that significantly more nonreciprocity could be observed on the reflection from antiferromagnetic surface by increasing the temperature.

Their study, however, concentrated on the classic Voigt geometry without considering the implications of hyperbolic behavior. In Fig. 8 we show the behavior of the reflectivity considering a higher temperature than what has been discussed thus far. In Fig. 8(a) we show the case when the anisotropy axis is parallel to the antiferromagnet's surface. In contrast to Fig. 6(a), here, the surface polariton is much wider in frequency, so that both upper and lower bulk bands are almost joined together in the reflection map. This increase in frequency width is due to the increased damping found at higher temperatures.

In Fig. 8(b) we show the effect of temperature on an antiferromagnetic crystal when the anisotropy axis is perpendicular to the surface. The path of the surface polariton itself is very similar to that shown in Fig. 6(b) for lower temperatures. However, here, it is again much wider, and the nonreciprocal behavior is much larger; that is, the contrast in reflectivity values for $+k_{\|}$and $-k_{\|}$is larger.

\section{DISCUSSION AND CONCLUSIONS}

Magnetic crystals were recently shown to be a good example of tunable hyperbolic media [31,32]. Here, we have investigated the fundamental behavior of magnetic polaritons in this class of materials in a geometry in which the easy axis of the antiferromagnet is at an arbitrary angle with respect to the surface. Because of the hyperbolic behavior we find new regions in $(\omega, k)$ where bulk polaritons are forbidden and where new surface polaritons exist. In the presence of an externally applied magnetic field the surface polaritons become strongly nonreciprocal.

At lower frequencies, 5-50 GHz, magnetic materials are used in a variety of signal-processing devices, including isolators, bandpass filters [33], band stop filters [34], and circulators [35]. Our findings indicate that antiferromagnets could play a similar role for signal processing in the $500-\mathrm{GHz}$ to $2-\mathrm{THz}$ frequency range $[36,37]$. Such terahertz magnons may also be used for sensing, working as an analog to the current plasmonic technologies $[38,39]$. We note in particular the nonreciprocity seen, for example, in Fig. 6(b), where there are some frequencies where a surface wave exists for $-k_{\|}$and no equivalent wave is present when propagation is reversed. Also, the group velocities for $+k_{\|}$are very different, with propagation along $+x$ having an average group velocity of about $0.0017 c$ and propagation along $-x$ having an average group velocity of about $0.01 c$.

It is important to point out that the new frequency gap where bulk waves are forbidden widens as the external field increases, as shown in Fig. 5(c). This is in substantial contrast to geometries previously studied, such as the Voigt geometry, where the forbidden gap regions become much narrower 

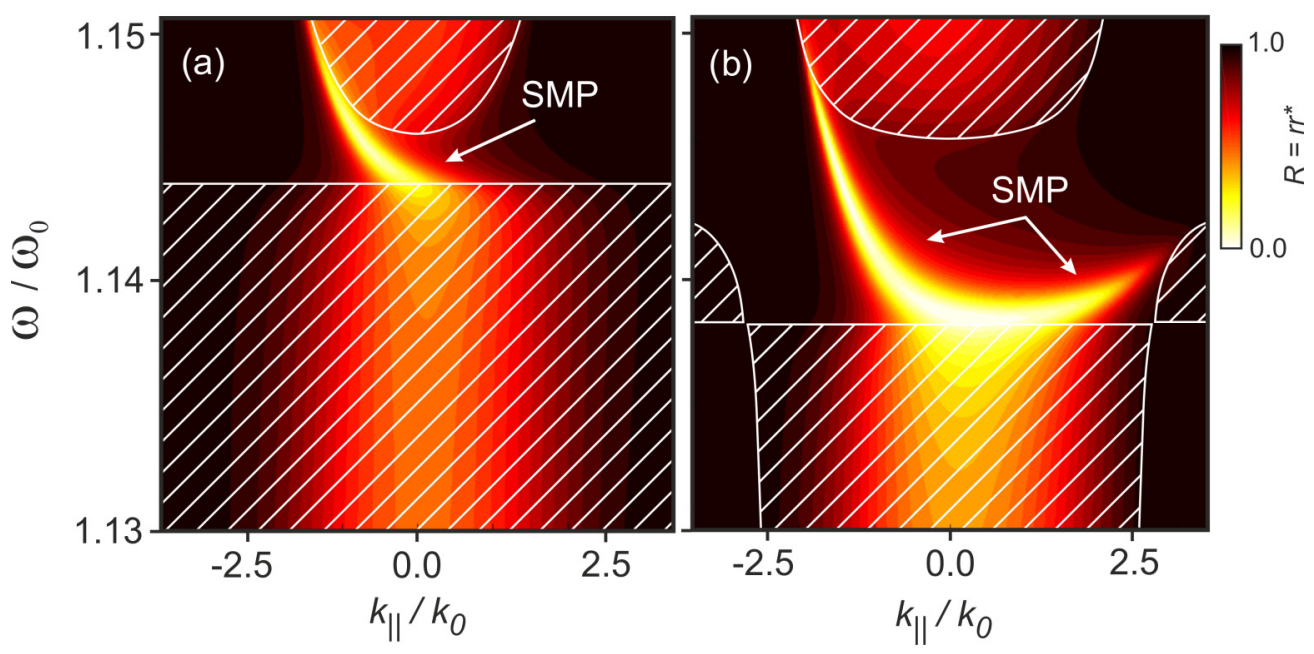

FIG. 8. Effects of temperature on the ATR and consequently, on the behaviour of the surface magnon-polaritons. Reflection maps calculated for a temperature $T=0.28 T_{N}$ and angle (a) $\varphi=0$ and (b) $\varphi=90^{\circ}$. Both plots are for an externally applied field $B_{0}=5.0 \mathrm{~T}$.

as the external field increases [16]. This means that effects associated with either the surface modes or the forbidden gap regions will not be weakened by an external field.

While we have concentrated on the nature of the surface magnon-polaritons, the rotation of the easy axis also has implications on the behavior of the bulk waves and novel effects such as negative refraction and slab lensing. This was recently investigated in natural dielectric media [25]. However, in magnetic crystals, not only is a similar behavior possible, but also tunability of these features could be achieved with externally applied fields.

Our findings may also have significant implications for a variety of other effects. An example of this is the GoosHänchen shift, where an incoming beam is laterally shifted upon reflection from the surface of an antiferromagnet. In this case, it was thought that the leakage of energy from the surface modes into the bulk bands, which is responsible for nonreciprocal behavior, would generate highly nonlinear lateral shifts [22]. More recently, however, surface polaritons were shown to be a direct mechanism by which lateral shifts can be enhanced [40]; thus, our discovery of new surface polaritons may be important here as well.

Finally, while we stress that the analysis and general principles presented in this paper can also be applied to other hyperbolic systems, the nonreciprocal behavior shown here is characteristic of gyrotropic media. These are only possible in media wherein the off-diagonal components of the permeability tensor couple directly with the magnetic field of the incident radiation.

\section{ACKNOWLEDGMENTS}

This work was financially supported by the Leverhulme Trust and the University of Glasgow through LKAS funds. We would also like to acknowledge useful discussions with N. R. Anderson and R. L. Stamps.

\section{APPENDIX A: ORIGINAL PERMEABILITY TENSOR}

The derivation of the full permeability tensor and its components has been detailed by Almeida and Mills in Ref. [21] for a canted antiferromagnet considering various configurations of the externally applied field, $\mathbf{B}_{\mathbf{0}}$. Here we quote only the relevant components for our particular geometry, depicted in Fig. 1(a), considering $\mathbf{B}_{\mathbf{0}}$ to be along the $y$ direction and $\varphi=0$. These components are:

$$
\begin{gathered}
\mu_{x x}=1+\frac{2 \mu_{0} \gamma^{2} M_{S} B_{0} \sin \alpha}{\omega_{\perp}^{2}-\omega^{2}}, \\
\mu_{z z}=1+\frac{2 \mu_{0} \gamma^{2} M_{S}\left(B_{0} \sin \alpha+B_{A} \cos 2 \alpha\right)}{\omega_{\perp}^{2}-\omega^{2}},
\end{gathered}
$$

while

$$
\mu_{x z}=-\mu_{x z}=-i \frac{2 \mu_{0} \gamma^{2} M_{S} \omega \sin \alpha}{\omega_{\perp}^{2}-\omega^{2}} .
$$

Here, $\alpha$ quantifies the canting angle and it is given by:

$$
\sin \alpha=\frac{B_{0}}{B_{A}+2 B_{E}} .
$$

Eqs. (A1) to (A3) can then be substituted into Eq. (4) to obtain the rotated tensor $\stackrel{\leftrightarrow}{\mu^{\prime}}(\omega)$.

The above components of the permeability tensor were derived under the assumption of no damping. However, such a phenomenon may generate relaxation of the precessional motion of the spins, which can change the linewidth of the resonant modes. In order to consider this, we introduce a phenomenological Bloch relaxation time $\tau$, which is directly proportional to $\omega_{0}$ being given by $\tau=1 / \Gamma$. The damping parameter $\Gamma$ can dramatically decrease the magnitude of the magnon-polariton resonance in the permeability tensor components [as seen in Figs. 1(b)-1(d)]. In order to consider the effects of damping in Eqs. (A1)-(A3), we replace $\omega \rightarrow$ $\omega+i \Gamma$.

\section{APPENDIX B: DISPERSION RELATIONS OF BULK AND SURFACE POLARITONS}

In order to find the dispersion of both bulk and surface polaritons, we employ Maxwell's curl equations, which have 
the form

$$
\begin{gathered}
\nabla \times \mathbf{E}=-\frac{\partial \mathbf{B}}{\partial t}, \\
\nabla \times \mathbf{H}=\frac{\partial \mathbf{D}}{\partial t},
\end{gathered}
$$

with the constitutive relations $\mathbf{B}=\mu_{0} \mu \mathbf{H}$ and $\mathbf{D}=\varepsilon_{0} \varepsilon \mathbf{E}$. These relate the electric and magnetic fields of the propagating radiation to the internal properties of the medium, given by $\mu$ and $\varepsilon$. In the antiferromagnet, $\mu=\overleftrightarrow{\mu^{\prime}}(\omega)$, given by Eq. (4), and in air $\mu=1$. Using these equations, we can look for polaritons propagating in the surface of the antiferromagnet with solution given by Eq. (6). Starting with Eq. (B1), we will find that

$$
\mathbf{B}(\vec{x}, t)=\left[\hat{x} \frac{i c}{\omega} \frac{\partial}{\partial z} E(x, z)-\hat{z} \frac{i c}{\omega} \frac{\partial}{\partial x} E(x, z)\right],
$$

which can be substituted into Eq. (B2) to find

$$
\begin{aligned}
& {\left[\frac{\partial^{2}}{\partial x^{2}} \mu_{x x}^{\prime}+\frac{\partial^{2}}{\partial z^{2}} \mu_{z z}^{\prime}+\frac{\partial}{\partial x \partial z}\left(\mu_{x z}^{\prime}\right.\right.} \\
& \left.\left.\quad+\mu_{z x}^{\prime}\right)+\frac{\omega^{2}}{c^{2}} \varepsilon_{3}\left(\mu_{x x}^{\prime} \mu_{z z}^{\prime}-\mu_{x z}^{\prime} \mu_{z x}^{\prime}\right)\right] E(x, z)=0 .
\end{aligned}
$$

This yields

$$
\begin{gathered}
-\beta^{2} \mu_{z z}^{\prime}+k_{\|}^{2} \mu_{x x}^{\prime}-i k_{\|} \beta\left(\mu_{x z}^{\prime}+\mu_{z x}^{\prime}\right) \\
=\frac{\omega^{2}}{c^{2}} \varepsilon_{3}\left(\mu_{x x}^{\prime} \mu_{z z}^{\prime}-\mu_{x z}^{\prime} \mu_{z x}^{\prime}\right) .
\end{gathered}
$$

The decay constant in the antiferromagnet $\beta$ is thus given by Eq. (B5). On the other hand, the equation for the decay constant in air, $\beta_{0}$, is much simpler, and it is given by

$$
\beta_{0}^{2}=k_{\|}^{2}-\frac{\omega^{2}}{c^{2}} \text {. }
$$

Note that Eqs. (B5) and (B6) can be converted into the bulk dispersion relation given in Sec. V [Eqs. (12) and (13)] by simply setting $\beta_{0}=i k_{\perp 2}$ and $\beta=i k_{\perp 3}$. In order to find a dispersion relation for surface polaritons, however, we need to match the tangential components of $H$ across the boundary, inside and outside of the material. These components can be found from Eq. (B3) to be

$$
H_{x}(x, t)=\frac{i \omega}{c}\left(\frac{\beta \mu_{z z}^{\prime} i k_{\|} \mu_{x z}^{\prime}}{\mu_{x x}^{\prime} \mu_{z z}^{\prime}-\mu_{x z}^{\prime} \mu_{z x}^{\prime}}\right) E_{y}(x, t)
$$

and

$$
H_{x}(x, t)=\frac{c}{i \omega} \beta_{0} E_{y}(x, t)
$$

in the antiferromagnet and in air, respectively. Matching these equations across the boundary, we obtain the implicit dispersion relation for surface polaritons given by Eq. (7).

\section{APPENDIX C: THEORY OF ATTENUATED TOTAL REFLECTION}

Since the derivation of the expressions for the reflectivity involves a sequence of steps familiar from ordinary optics $[15,30]$, we only outline the procedure. We assume the radiation propagates with an $\mathbf{E}$ field of the form

$$
\mathbf{E}(\vec{x}, t)=\hat{y} e^{i k_{\|} x-i \omega t}\left(a_{n} e^{i k_{\perp n} z}+b_{n} e^{-i k_{\perp n} z}\right),
$$

where $a$ and $b$ represent the amplitude of the field propagating up and down across each layer and the subscript $n$ is either (1) for a prism, (2) for air, or (3) for an antiferromagnet. Note that since we are interested in the case of a semi-infinite antiferromagnet and there are no waves reflected within that layer, $b_{3}=0$.

We then match the boundary conditions of tangential $\mathbf{H}$ and tangential $\mathbf{E}$, which yields a system of equations which can be written as the following matrix:x

$$
\left.\begin{array}{c}
0 \\
0 \\
\frac{k_{\perp 3} \mu_{z}^{\prime}+k_{\|} \mu_{x z}^{\prime}}{k_{\perp 2}\left(\mu_{x x}^{\prime} \mu_{z z}^{\prime}-\mu_{x z}^{\prime} \mu_{z x}^{\prime}\right)}
\end{array}\right]\left[\begin{array}{c}
b_{1} \\
a_{2} \\
b_{2} \\
a_{3}
\end{array}\right]=\left[\begin{array}{c}
a_{1} e^{-i k_{\perp 1} d} \\
a_{1} e^{-i k_{\perp 1} d} \\
0 \\
0
\end{array}\right] .
$$

The reflection coefficient can be found by solving $b_{1} / a_{1}$. The solution of Eq. (C2) can then be used to construct the reflection maps shown throughout this paper.

[1] D. R. Smith, W. J. Padilla, D. C. Vier, S. C. Nemat-Nasser, and S. Schultz, Composite Medium with Simultaneously Negative Permeability and Permittivity, Phys. Rev. Lett. 84, 4184 (2000).

[2] A. Boltasseva and H. A. Atwater, Low-loss plasmonic metamaterials, Science 331, 290 (2011).

[3] N. V. Ilin, I. G. Kondratiev, and A. I. Smirnov, True surface waves guided by metamaterials, Bull. Russ. Acad. Sci.: Phys. 72, 118 (2008).

[4] R. C. Owen, K. S. Murphy, K. G. Hoke, T. J. Morgan, B. L. Johnson, and J. M. Leger, Detection of guided-wave plasmon polariton modes in a high-index dielectric MIM structure, J. Appl. Phys. 122, 123101 (2017).

[5] F. Peragut, L. Cerruti, A. Baranov, J. P. Hugonin, T. Taliercio, Y. De Wilde, and J. J. Greffet, Hyperbolic metamaterials and surface plasmon polaritons, Optica 4, 1409 (2017).

[6] C. J. Zapata-Rodríguez, J. J. Miret, S. Vuković, and M. R. Belić, Engineered surface waves in hyperbolic metamaterials, Opt. Express 21, 19113 (2013).

[7] D. N. Basov, M. M. Fogler, and F. J. García de Abajo, Polaritons in van der Waals materials, Science 354, aag1992 (2016). 
[8] A. A. Govyadinov, A. Konečná, A. Chuvilin, S. Vélez, I. Dolado, A. Y Nikitin, S. Lopatin, F. Casanova, L. E Hueso, and J. Aizpurua, Probing low-energy hyperbolic polaritons in van der Waals crystals with an electron microscope, Nat. Commun. 8, 95 (2017).

[9] T. Low, A. Chaves, J. D. Caldwell, A. Kumar, N. X. Fang, P. Avouris, T. F. Heinz, F. Guinea, L. Martin-Moreno, and F. Koppens, Polaritons in layered two-dimensional materials, Nat. Mater. 16, 182 (2017).

[10] D. Wintz, K. Chaudhary, K. Wang, L. A. Jauregui, A. Ambrosio, M. Tamagnone, A. Y. Zhu, R. C. Devlin, J. D. Crossno, K. Pistunova, K. Watanabe, T. Taniguchi, P. Kim, and F. Capasso, Guided modes of anisotropic van der Waals materials investigated by near-field scanning optical microscopy, ACS Photonics 5, 1196 (2018).

[11] W.-L. Gao, F.-Z. Fang, Y.-M. Liu, and S. Zhang, Chiral surface waves supported by biaxial hyperbolic metamaterials, Light: Sci. Appl. 4, e328 (2015).

[12] D. R. Smith and D. Schurig, Electromagnetic Wave Propagation in Media with Indefinite Permittivity and Permeability Tensors, Phys. Rev. Lett. 90, 077405 (2003).

[13] D. L. Mills, and E. Burstein, Polaritons: The electromagnetic modes of media, Rep. Prog. Phys. 37, 817 (1974).

[14] R. E. Camley, Long-Wavelength Surface Spin Waves on Antiferromagnets, Phys. Rev. Lett. 45, 283 (1980).

[15] R. E. Camley, and D. L. Mills, Surface polaritons on uniaxial antiferromagnets, Phys. Rev. B 26, 1280 (1982).

[16] R. E. Camley, Nonreciprocal surface waves, Surf. Sci. Rep. 7, 103 (1987).

[17] J.-X. Ta, Y. Han, and C. Lan, The comparative study of bulk magneto-phonon and magnetic polaritons of lateral antiferromagnetic superlattices for potential THz applications, J. Magn. Magn. Mater. 399, 245 (2016).

[18] R. Macêdo and T. Dumelow, Tunable all-angle negative refraction using antiferromagnets, Phys. Rev. B 89, 035135 (2014).

[19] R. Macêdo, T. Dumelow, and R. L. Stamps, Tunable focusing in natural hyperbolic magnetic media, ACS Photonics 3, 1670 (2016).

[20] F. Keffer and C. Kittel, Theory of antiferromagnetic resonance, Phys. Rev. 85, 329 (1952).

[21] N. S. Almeida and D. L. Mills, Dynamical response of antiferromagnets in an oblique magnetic field: Application to surface magnons, Phys. Rev. B 37, 3400 (1988).

[22] R. Macêdo, R. L. Stamps, and T. Dumelow, Spin canting induced nonreciprocal Goos-Hänchen shifts, Opt. Express 22, 28467 (2014).

[23] L. Remer, B. Lüthi, H. Sauer, R. Geick, and R. E. Camley, Nonreciprocal Optical Reflection of the Uniaxial Antiferromagnet $\mathrm{MnF}_{2}$, Phys. Rev. Lett. 56, 2752 (1986).

[24] M. A. K. Othman, C. Guclu, and F. Capolino, Graphene-based tunable hyperbolic metamaterials and enhanced near-field absorption, Opt. Express 21, 7614 (2013).

[25] R. Macêdo, T. Dumelow, R. E. Camley, and R. L. Stamps, Oriented asymmetric wave propagation and refrac- tion bending in hyperbolic media, ACS Photonics 5, 5086 (2018).

[26] T. Xu and H. J. Lezec, Visible-frequency asymmetric transmission devices incorporating a hyperbolic metamaterial, Nat. Commun. 5, 4141 (2014).

[27] M. R. F. Jensen, S. A. Feiven, T. J. Parker, and R. E. Camley, Experimental determination of magnetic polariton dispersion curves in $\mathrm{FeF}_{2}$, Phys. Rev. B 55, 2745 (1997).

[28] M. R. F. Jensen, T. J. Parker, K. Abraha, and D. R. Tilley, Experimental Observation of Magnetic Surface Polaritons in $\mathrm{FeF}_{2}$ by Attenuated Total Reflection, Phys. Rev. Lett. 75, 3756 (1995).

[29] R. Macêdo, K. L. Livesey, and R. E. Camley, Using magnetic hyperbolic metamaterials as high frequency tunable filters, Appl. Phys. Lett. 113, 121104 (2018).

[30] N. R. Anderson, and R. E. Camley, Attenuated total reflection study of bulk and surface polaritons in antiferromagnets and hexagonal ferrites: Propagation at arbitrary angles, J. Appl. Phys. 113, 013904 (2013).

[31] S. S. Kruk, Z. J. Wong, E. Pshenay-Severin, K. O’brien, D. N. Neshev, Y. S. Kivshar, and X. Zhang, Magnetic hyperbolic optical metamaterials, Nat. Commun. 7, 11329 (2016).

[32] C. Lan, K. Bi, J. Zhou, and B. Li, Experimental demonstration of hyperbolic property in conventional material-ferrite, Appl. Phys. Lett. 107, 211112 (2015).

[33] C. S. Tsai, G. Qiu, H. Gao, L. W. Yang, G. P. Li, S. A. Nikitov, and Y. Gulyaev, Tunable wideband microwave bandstop and band-pass filters using YIG/GGG-GAAS layer structures, IEEE Trans. Magn. 41, 3568 (2005).

[34] Q. Wang, L. Zeng, M. Lei, and K. Bi, Tunable metamaterial bandstop filter based on ferromagnetic resonance, AIP Adv. 5, 077145 (2015).

[35] A. Saib, M. Darques, L. Piraux, D. Vanhoenacker-Janvier, and I. Huynen, Unbiased microwave circulator based on ferromagnetic nanowires arrays of tunable magnetization state, J. Phys. D 38, 2759 (2005).

[36] K. Grishunin, T. Huisman, G. Li, E. Mishina, T. Rasing, A. V. Kimel, K. Zhang, Z. Jin, S. Cao, W. Ren, G.-H. Ma, and R. V. Mikhaylovskiy, Terahertz magnon-polaritons in $\mathrm{TmFeO}_{3}$, ACS Photonics 5, 1375 (2018).

[37] M. Shalaby, M. Peccianti, Y. Ozturk, and R. Morandotti, A magnetic non-reciprocal isolator for broadband terahertz operation, Nat. Commun. 4, 1558 (2013).

[38] S. M. Hanham, and S. A. Maier, Terahertz plasmonic surfaces for sensing, in Active Plasmonics and Tuneable Plasmonic Metamaterials (John Wiley \& Sons, Ltd, Hoboken, New Jersey, 2013), pp. i-xviii.

[39] S. M. Hanham, M. Navarro-Cía, B. Ng, H. Aouani, M. Rahmani, N. Klein, and S. A. Maier, Exploiting plasmonics for THz and infrared sensing, Proc. SPIE 9102, 91020J (2014).

[40] V. B. Silva and T. Dumelow, Surface mode enhancement of the Goos-Hänchen shift in direct reflection off antiferromagnets, Phys. Rev. B 97, 235158 (2018). 